\title{
Effect of 802.11 Adaptive Exponential Backoffs on the Fluidity of Downlink Flows in Mesh Networks
}

\author{
Adel Aziz \\ EPFL \\ Lausanne, Switzerland \\ adel.aziz@epfl.ch
}

\author{
Roger Karrer \\ Deutsche Telekom Laboratories \\ Berlin, Germany \\ roger.karrer@telekom.de
}

\author{
Patrick Thiran \\ EPFL \\ Lausanne, Switzerland \\ patrick.thiran@epfl.ch
}

\begin{abstract}
Efficient multihop traffic management is a need for successful Wireless Mesh Networks (WMNs) deployment. Using an analogy with fluid mechanism, we classify a flow as laminar if the packets flow smoothly from the Wired Access Point (WAP) over the mesh network, and as turbulent otherwise. We identify a particular but frequent collision scenario, which sets the flow to be turbulent, resulting in a strongly reduced downlink endto-end throughput. We show that the exponential backoff mechanism in an 802.11 WMN is responsible for this problem and suggest a modification of the current exponential backoff policy of 802.11 for WMNs. We support these findings both with simulations and real measurements on a testbed infrastructure.
\end{abstract}

\section{INTRODUCTION}

The deployment of wireless mesh networks that cover large areas such as entire cities is rapidly increasing [1], [2], [3], [4]. This deployment is astonishing because business cases are far from certain and because our knowledge about building and operating mesh networks efficiently is still in its infancy. In particular the backhaul of a mesh network where data is forwarded over multiple hops from and to a wired mesh node and which therefore provides the key cost savings for mesh networks frequently shows dismal single-digit throughputs [7], [8].

The culprit has been identified in many previous studies: the random access mechanisms of the 802.11 MAC are not efficient in backhaul networks. While the random access provides a fair access for randomly distributed nodes in a given area, it is far from efficient for the particular requirements of a wireless backhaul. The backhaul should forward flows in a 'laminar' way, i.e. packets should smoothly be passed from one node to the next one, in the same way traffic lights should sequentially show green lights, rather than creating a bumpy 'turbulent' traffic pattern due to unsynchronized traffic lights. We argue that such a laminar flow behavior improves the overall network throughput and provides better per-flow end-to-end behavior, such as lower delays and lower jitter.

Toward this objective, this paper makes three contributions. First, we provide evidence that turbulent behavior occurs in backhaul networks with 802.11 MACs. With a simple example, we show that the queues of some nodes rapidly build up whereas other nodes have empty queues. Second, we propose a solution for the above problem: (i) replace the exponential backoff policy of 802.11 by a fixed contention window and (ii) increase the retry limit of retransmitting packets. We show with analytical and simulation results that the total throughput of a mesh backhaul can be increased by $82 \%$ in a linear topology. Third, we experimentally evaluate our proposition in the Magnets indoor testbed. The measurements confirm that current 802.11 MACs create turbulent flow patterns and that our modifications lead to a laminar behavior. Thus, end-to-end throughput and total capacity increase. This evaluation also emphasizes that the benefits can be achieved by simple modifications of 802.11 parameters but without fundamentally changing the 802.11 protocol.

This paper is organized as follows. Section II provides background on the problem statement of multihop data forwarding, the failure of 802.11, our concept and related work. Section III verifies our concept with simulations, and Section IV presents our experimental evaluation in an indoor mesh testbed. We then conclude in Section VI.

\section{802.11 IN MULTIHOP BACKHAUL NETWORKS}

This section provides background on the problem of multihop flow behavior and its causes.

\section{A. Problem statement}

Wireless mesh networks consist of two parts: an access part that provides connectivity to the user and a backhaul network that transports data over multiple wireless hops called transit access points (TAPs) from and to a Wired Access Point (WAP) that is equipped 


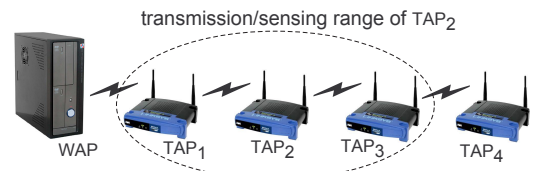

(a) 1-ary topology: De-centralized WAP

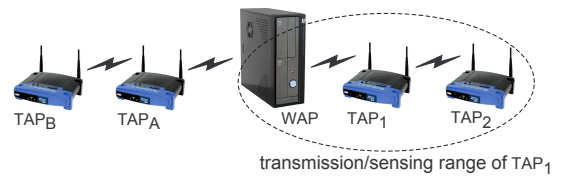

(b) 2-ary topology: Centralized WAP

Fig. 1. Illustration of the mesh networks studied.

with a fixed network line. The logical topology of the backhaul is typically arranged as a k-ary tree, with the WAP as the root and the access points that connect to the users as leafs. For simplicity reasons, we initially consider only linear topologies $(k \leq 2)$, as depicted in Figure 1. Due to its primordial role of connecting the backhaul to external networks such as the Internet, the WAP is the node through which all the traffic flows. Therefore, it is likely to be the bottleneck of the network. Moreover, we focus on downstream traffic, i.e. traffic from the WAP to the users because applications such as Web, multimedia streaming or P2P systems typically have larger downstream demands than upstream ones.

\section{B. Objective}

We define the efficiency of the backhaul network by two metrics. First, the total achieved throughput should be maximized, ideally matching the network capacity. Second, the end-to-end performance of each flow should be maximized. In particular, delays should be low and have low variations (for TCP as well as VoIP), and packet loss should be minimized.

We argue that these objectives are best achieved when the flows through the backhaul are laminar.

Definition 1 (Laminar flow): Laminar flows are characterized by a smooth propagation of packets through the network, where every packet only spends a negligible time in any TAP's buffer. They satisfy the following condition on the buffers $B_{i}$ :

$$
\operatorname{Prob}\left(B_{i} \text { full }\right) \approx 0 \quad \forall i \neq W A P
$$

The opposite of laminar flows are turbulent flows:

Definition 2 (Turbulent flow): Turbulent flows are characterized by packets spending a significant amount of time in the buffer of TAPs.

$$
\operatorname{Prob}\left(B_{i} \text { full }\right) \gg 0 \quad \text { for at least one } i \neq W A P
$$

When flows traverse multiple hops, this queuing delay creates perturbation in the flow propagation.
Furthermore, we will see for the scenario described in the next subsection, that it is the first TAP that creates turbulent flows, so that (2) is verified for $i=1$.

To motivate our argument why laminar flows are desirable, consider the analogy of vehicular traffic. Along a road, traffic passes smoothly through if the traffic lights are shifted in sequence. Under ideal conditions, a car can cruise at constant speed. Cars only have to wait at the first traffic light. Along the road, no car ever has to wait at a traffic light. Nor do cars have to break and therefore no collisions occur.

Reverting back this behavior to a backhaul network, laminar flows ideally have a constant delay through the mesh network and therefore improve the stability of TCP-based flows as well as the quality of delay-sensitive applications such as VoIP and multimedia streaming. Moreover, having no packets waiting at the TAPs incurs no collisions that might reduce the overall network throughput.

\section{Failure of 802.11}

Unfortunately the current 802.11 standard [5], which has been designed for a fair resource sharing in singlecell communication, is far from achieving a laminar flow behavior over a multihop backhaul network. To understand this statement, we briefly describe the basic mechanisms in 802.11. A node that wants to transmit data senses the medium using RTS/CTS. If the physical layer does not detect activity on the link and the Network Allocator Vector (NAV) counter is null, the medium is considered idle and the node starts transmitting. Otherwise, the channel is considered busy and the node starts to backoff when the channel returns to idle.

The backoff mechanism consists of a counter that is initially uniformly selected in the interval $[0 ; c w]$, where the contention window $\mathrm{cw}$ has a value between $C W_{\min }$ (31 for $802.11 \mathrm{~b}$ and 15 for $802.11 \mathrm{a} / \mathrm{g}$ ) and $C W_{\max }$ (1023). The exact $c w$ value is obtained by an exponential increase mechanism, i.e. $c w$ is initialized at $C W_{\min }$ and it is doubled as long as the packet experiences a collision till reaching the $C W_{\max }$ limit. Finally, $c w$ is reset to $C W_{\min }$ after a successful transmission of the packet. The backoff counter consists of slots of $20 \mu \mathrm{s}$ and is decremented as long as the channel is sensed idle and remains frozen if it is not the case. Eventually, when the counter reaches zero, the node sends the message over the medium following the RTS-CTS mechanism.

We now illustrate that these mechanisms lead to turbulent behavior in multihop backhaul networks. Figure 2(a) depicts the transmissions as a function of the time, 


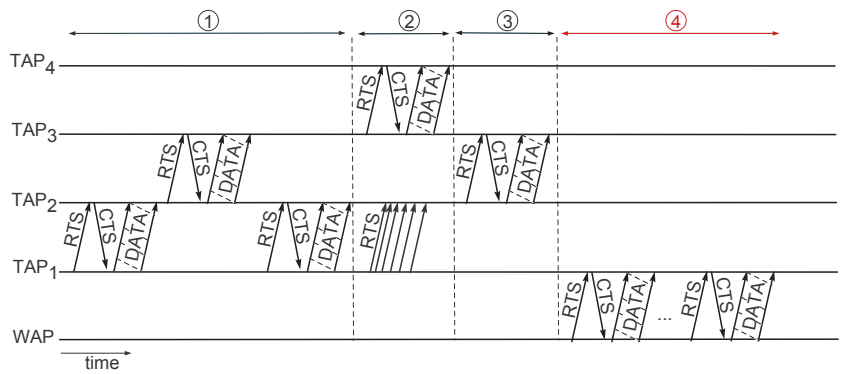

(a) Link activity (ACK messages omitted).

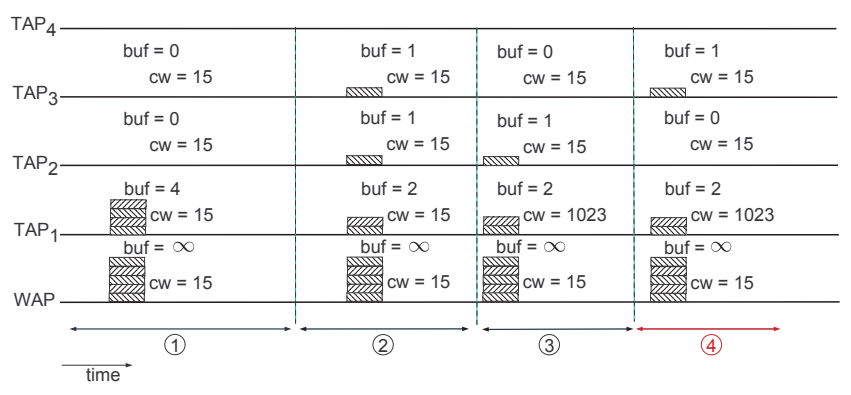

(b) Buffer size and $c w$ evolution at the beginning of each phase.

Fig. 2. Illustration of the perturbation creation due to the exponential backoff of MAC 802.11.

whereas Figure 2(b) shows the corresponding queues and the values of the contention window $c w$ for the topology depicted in Figure 1(a). We assume that the WAP has always traffic to send, so that its buffer is full (which we denote by $\infty$ ), and that $T A P_{1}$ has already 4 packets buffered. The build-up of the queues that lead to a turbulent behavior can be separated into 4 phases:

1) Phase 1: Packets are sent from $T A P_{1}$ to $T A P_{2}$ and $T A P_{3}$. At the end of this phase, each buffer contains at least one packet.

2) Phase 2: $T A P_{3}$ transmits a packet to $T A P_{4}$. $T A P_{1}$ is out of the sensing range of $T A P_{3}$ : it is therefore unaware of this transmission, and sends unsuccessful RTS. These RTS make $W A P$ set its NAV properly, and increase the contention window of $T A P_{1}$ up to its maximal value $C W_{\max }=1023$.

3) Phase 3: $T A P_{2}$ transmits a packet to $T A P_{3}$. As the WAP is unaware of this transmission, its backoff counter is not frozen and will eventually reach 0 . On the other hand, the NAV of $T A P_{1}$ is set by the RTS, which prevents it to decrement its contention window. Therefore, the contention window of $T A P_{1}$ remains at a high value (around $\left.C W_{\max }\right)$.

4) Phase 4: The transmission of $T A P_{2}$ terminates. $T A P_{1}$ and $W A P$ still have packets to send and compete for the channel. However their competi- tion is not fair, because the contention window of $T A P_{1}$ is much larger than that of the WAP (1023 compared to 31 for 802.11 b (or 15 for $802.11 \mathrm{a}$ ) in our example, a ratio factor of 32 (or even 64)!). This unfair advantage will make $W A P$ win the channel many times in a row. As a result, the buffer of $T A P_{1}$ builds up. This increase leads to the perturbation in the fluidity of the data flow.

\section{Proposed Solution}

To solve the buffer building-up issue, the consequences of the physical limitation should be reduced by preventing an unfair competition for the medium between $T A P_{1}$ and $W A P$ due to $\mathrm{cw}$. We argue that a possible solution to reach this goal is achievable with 2 modifications within 802.11:

- The exponential backoff mechanism is disabled and replaced by a fixed value for $c w$ to ensure that unfair competition among the WAP/TAPs does not occur independently of the communication taking place previously.

- The short retry limit value which sets the maximum number of attempted transmissions before dropping a packet should be increased. Indeed, when the exponential backoff mechanism is disabled, the time needed to reach the retry limit decreases. An increase in the retry limit therefore avoids that packets are dropped too early. Packets that have left the WAP should not be dropped by any of the TAPs.

These two modifications require just changes in the parameters of 802.11 and are therefore easy to implement. Furthermore, even though the total number of collisions may increase due to this more aggressive policy, the end-to-end performances remain improved. The intuition behind these findings is obtained by considering the packet flow and noticing that when a collision happens between 2 links, only the downstream link is penalized but not the upstream one. This phenomenon therefore acts similarly to a virtual back-pressure that will promote laminar flows pushing packets further in the network and then improves the end-to end performances

\section{THROUGHPUT ANALYSIS}

This section verifies the above findings with simulations and assesses the impact on the throughput.

\section{A. Setup}

We set up a linear (1-ary and 2-ary) topology that matches the topology shown in Figure 1. We simulate a topology of $n$ nodes per branch, where $n$ is varied 
from 4 to 20 TAPs, not including the WAP. The distance among the TAPs is chosen such that the sensing and the transmission range include the direct neighbors, but not the neighbors that are 2 hops away. The link capacity is set to $1 \mathrm{Mb} / \mathrm{s}$ and we use packet sizes of 1500 Bytes.

Given these values and neglecting the effect of DIFS and SIFS, the theoretical single-link throughput can be calculated as

$$
\frac{P A Y L O A D}{D A T A+R T S+C T S+A C K+t_{B A C K O F F} * b w} * b w
$$$$
\text { With } P A Y L O A D=1500 \text { Bytes, DATA= }
$$
1572 Bytes $(1500 B+6 B$ (PLC header) $+34 B$ (MAC header $)+24 B$ (IP header $)+8 B($ UDP header $)), R T S=$ 44 Bytes, $C T S=A C K=38$ Bytes, $t_{B A C K O F F} * b w=$ 40 Bytes and bandwidth bw $=1 / 8 * 10^{6} \mathrm{~B} / \mathrm{s}$, we get a theoretical single-link throughput of $108.26 \mathrm{kB} / \mathrm{s}$ $(866 \mathrm{~kb} / \mathrm{s})$.

For multihop topologies, the theoretical maximal throughput can be computed considering the maximal spatial reuse of a k-ary topology. Assuming the standard 2-hop collision model, i.e. 2 links can only be active simultaneously if they are separated by 2 other intermediate links, the throughput for a 1-ary topology (respectively, 2-ary topology) is easily computed to be one third (respectively, one half) of the capacity [9]. Therefore, the upper-bound on the throughput performance is $36.09 \mathrm{kB} / \mathrm{s}(288 \mathrm{~kb} / \mathrm{s})$ for a 1-ary topology and $54.13 k B / s(433 k b / s)$ for a 2-ary topology.

\section{B. Simulation Results}

Figure 3 shows the impact of the proposed modifications to the 802.11 parameter values as a function of the number of nodes for 1-ary topologies (Figure 3(a)) and 2-ary topologies (Figure 3(b)). The 3 lines denote the throughput derived from our analytical analysis, with exponential backoff (standard) and with our proposed solution of fixed contention window $c w$ and significantly increased retry limit (1000). Such an extreme value for the retry limit is motivated by the fact that currently most flows in the Internet are TCP flows. Dropping a packet implies that the packet needs to be retransmitted over the entire end-to-end path. Such a retransmission uses significantly more resources than a local retransmission.

In Figure 3(a), we first note that the standard 802.11 with exponential backoff achieves a dismal $44 \%$ of the theoretical throughput for $n=20$ nodes. Moreover, significant throughput degradations are already visible for multihop networks of size 4 . In contrast, with a fixed $c w$ and increased retry limit, the throughput increases to $79 \%$ of the theoretical achievable throughput even for network sizes of $n=20$ nodes. Furthermore,

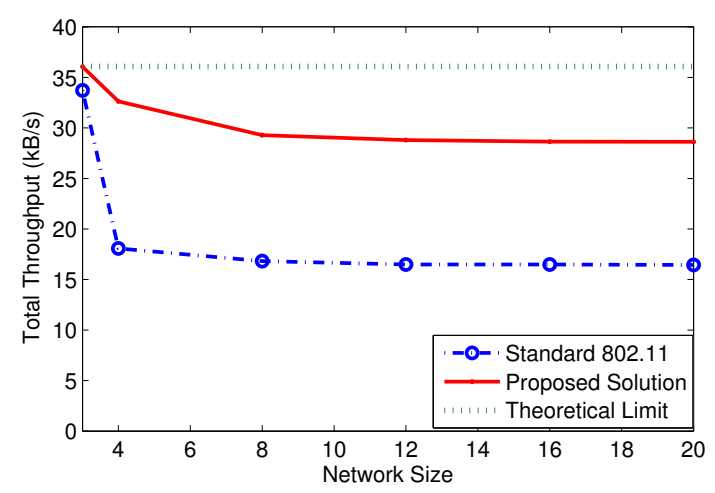

(a) 1-ary topology.

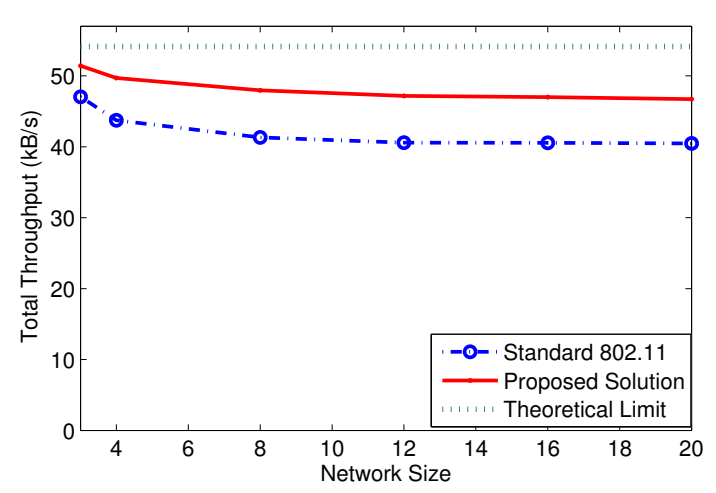

(b) 2-ary topology

Fig. 3. Performance gain achievable by removing the exponential backoff policy and increasing the short retry limit.

for small topologies of 4-hops our proposed solution achieves performance as high as $90 \%$ of the theoretical maximum. Thus, our proposed scheme almost doubles the throughput compared to 802.11.

For 2-ary topologies, the results in relative terms are comparable to the 1 -ary ones. In particular, our proposed solution achieves $87 \%$ of the theoretical limit for $n=20$ nodes, while standard 802.11 only achieves $70 \%$. These results confirm that the modifications of the 802.11 parameter values have a significant impact on the effective throughput of a multihop wireless network.

\section{EXPERIMENTAL EVALUATION}

This section verifies the proposed modifications for 802.11 with measurements in a wireless testbed. We emphasize here also that the modifications were readily "implemented" because we only adjusted some parameter values but did not need to change the MAC layer protocol.

\section{A. Testbed setup}

We perform our measurements in the indoor mesh testbed of the Magnets project [10]. We deployed two 5- 


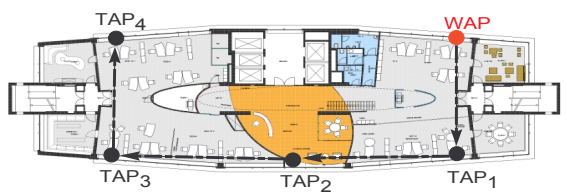

Fig. 4. Topology 1: all nodes are on the same floor.

hop topologies. In one topology, all nodes were deployed on the same floor of the office building, as depicted in Figure 4. In the second setup, we deployed the nodes on adjacent floors. Both topologies allow us to closely match the interference model of our linear scenario in Figure 1 because the metal and concrete structure of the building prevent interference over multiple hops, i.e. nodes that are 2 hops apart are not within sensing range.

During the deployment and measurements on the testbed, we made similar observations as in [16] concerning the significant performance variability to millimeter changes of the position or direction of the antenna. Such variations do not impact our results as we maintained the location strictly unchanged during the simulation rounds.

The nodes consists of Routerboards 532 that are equipped with one Atheros-based $802.11 \mathrm{a} / \mathrm{b} / \mathrm{g}$ card. We use the 802.11a mode to avoid interference from other networks and fix the channel to $5.32 \mathrm{GHz}$. For the same reason, we run the experiments at night. The cards are connected to $3 \mathrm{~dB}$ indoor omni-directional antennas. The boards run the Kamikaze version of OpenWRT 2.6.21.5 with the MadWifi driver. At the network layer, we use fixed routing to exclude routing messages and potential problems from route changes.

As traffic source and sink, we use 2 Linux-based PCs. On these PCs we run iperf [21]. The sender is connected via an Ethernet connection to the WAP, the receiver is also connected via a fixed line to TAP 4 . An experiment consists of multiple runs with different values for $C W_{\min }$. For each run, UDP traffic is generated at a rate of $10 \mathrm{Mb} / \mathrm{s}$. We ensured that this rate is far above the network capacity and that therefore the WAP always has packets in its buffer to achieve the conditions described in Figure 2. Each run lasts for 150 seconds. In our evaluation, we ignore the first 50 seconds to avoid initial fluctuations and collect stationary regime results.

For each run, we log the achieved throughput and average it for each second. The 100 obtained values are then used to compute an average over 100 seconds together with confidence intervals obtained using the normality assumption.

The results present the comparison of standard 802.11

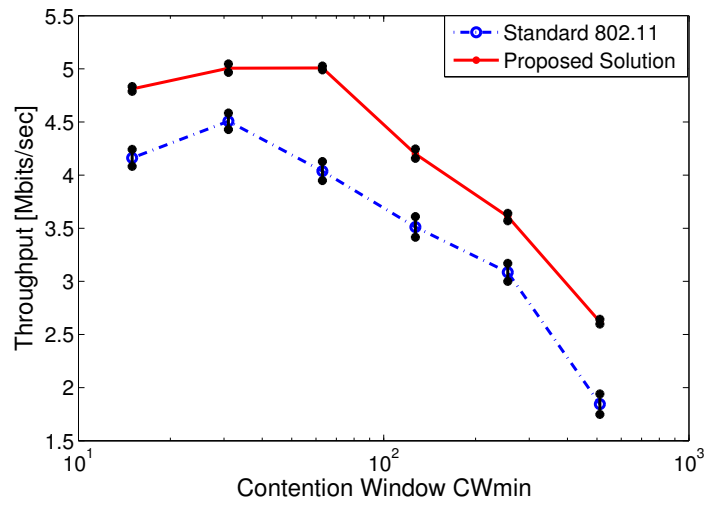

(a) Topology 1

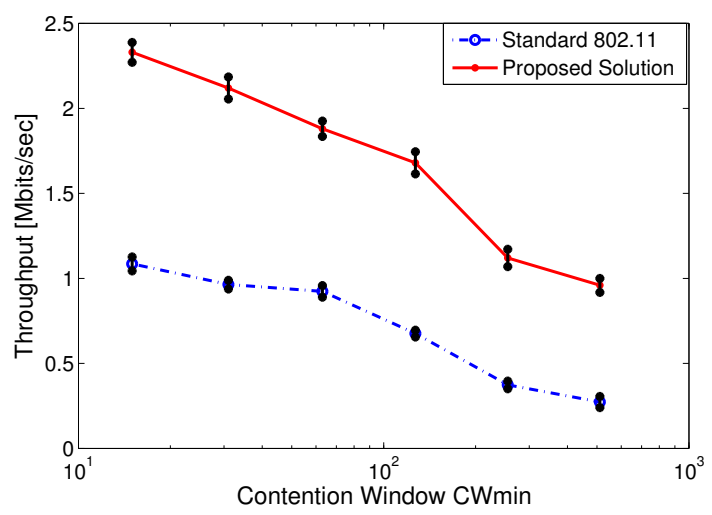

(b) Topology 2

Fig. 5. 5-hop throughput as a function of $c w$ values.

with our proposed solution for different value of $C W_{\text {min }}$. By standard 802.11, we consider keeping the exponential backoff with $C W_{\max }=1023$ and all the parameter of 802.11 constant while only varying $C W_{\min }$. On the other hand, as defined in Section 2.4, our proposed solution consist in fixing the contention window at $c w=C W_{\min }$ and increasing the retry limit to 1000 to match our simulation model.

\section{B. Throughput Measurement Results}

Figure 5 shows the multihop throughput obtained in our testbed, as a function of the value of $C W_{\min }$. The $\mathrm{x}$-axis is logarithmically scaled because the values are typically powers of 2 . First, considering the lines in Figure 5(a), we note that the throughput rapidly degrades as a function of the value of $C W_{\min }$ after some initial increase. The initial increase can be explained by the reduction of the collision probability due to the $c w$ increase. Second, comparing 802.11 against our proposed solution, we note a difference of roughly $0.5 \mathrm{Mb} / \mathrm{s}$, or between $10 \%$ and $60 \%$ in relative terms.

For the second topology, the throughputs shown in Figure 5(b) shows three significant differences compared 

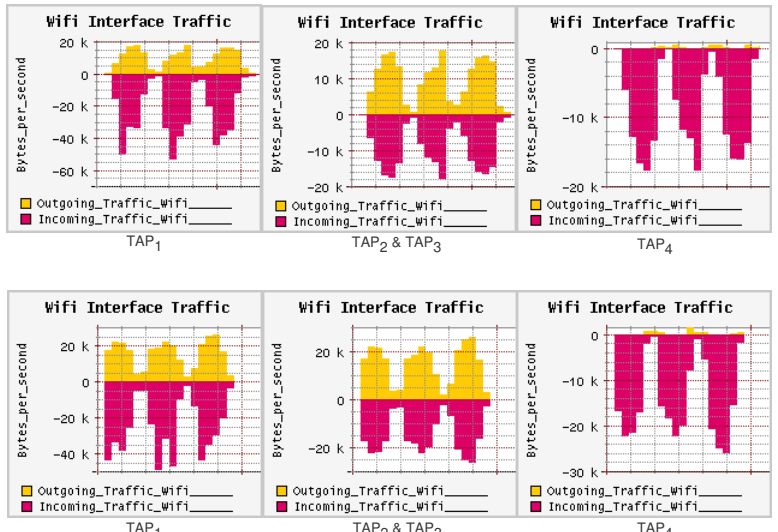

(a) Detailled I/O statistics for 3 rounds of: standard 802.11 (top) and our proposed modifications (bottom).

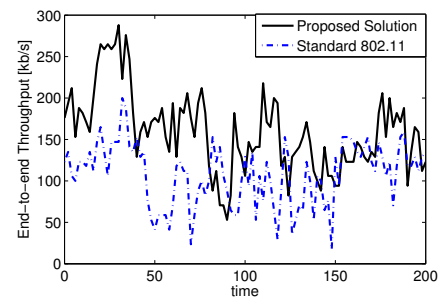

(b) End-to-end throughput.

Fig. 6. Measurements results of saturated UDP traffic.

to the results in Figure 5(a). First, the throughput is significantly lower. This low throughput can be attributed to the larger distances and in particular the ceilings in the buildings that damp the signal. Therefore, the achieved rates are more than 50\% lower than those of the previous experiment. Second, we do not see the initial increase in the throughput for low values of $C W_{\min }$. These findings indicate that an optimal $C W_{\min }$ value is a topology dependent parameter. Finally, the difference between standard 802.11 and our solution is more exposed. Our solution outperforms standard 802.11 by more than 1 $\mathrm{Mb} / \mathrm{s}$, a net improvement of more than $100 \%$ !

\section{Additional Measurement Results}

In order to confirm and extend the measurement presented in Section IV-B by more detailed statistics, we deployed an additional small-scale 5-nodes testbed satisfying our required topology and with setup details described in [6]. Using this new setup, we present in Figure 6 the results obtained for saturated UDP traffic and in Figure 7 the ones for the TCP scenario.

These additional measurements for the UDP scenario allow confirming the nature of $T A P_{1}$ as bottleneck of the system. Indeed as $T A P_{1}, T A P_{2}$ and $T A P_{3}$ are neither source nor sink of traffic, they should optimally forward all the traffic they receive and therefore have
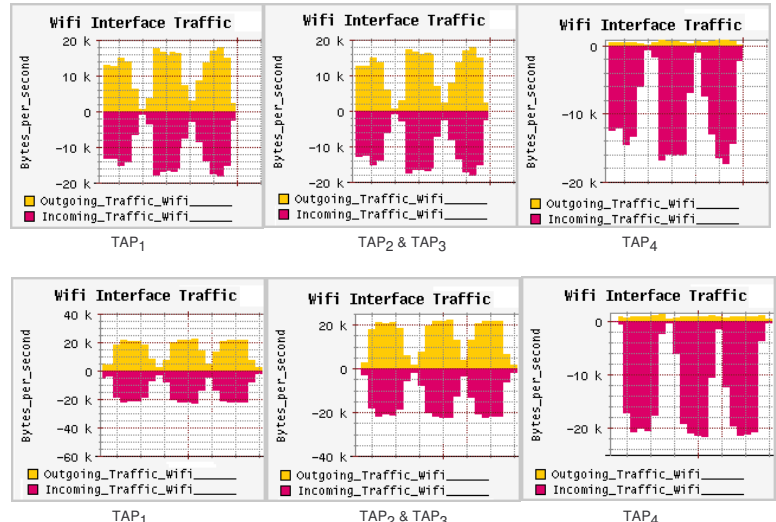

(a) Detailled I/O statistics for 3 rounds of: standard 802.11 (top) and our proposed modifications (bottom).

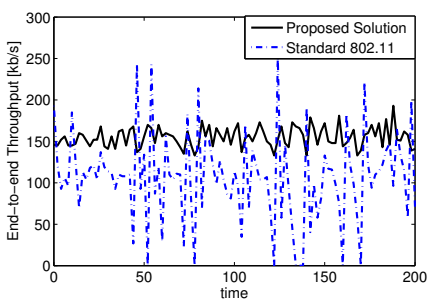

(b) End-to-end throughput.

Fig. 7. Measurements results of TCP traffic.

an equivalent quantity of incoming and outgoing traffic. However this equivalence is only satisfied for $T A P_{2}$ and $T A P_{3}$, but not for $T A P_{1}$, where the incoming traffic is significantly higher than the outgoing one, which indicates that the buffer builds up at $T A P_{1}$ and packets are therefore dropped. As expected by our analysis, our proposition of fixing the contention window significantly improves the situation at $T A P_{1}$. Therefore, even though our solution does not completely solve the problem, it significantly improves the situation, which is reflected by an increased end-to-end throughput.

Concerning the TCP throughput, it is interesting to first notice that the unbalanced situation on $T A P_{1}$ 's $\mathrm{I} / \mathrm{O}$ share does not happen due to the TCP feedback that performs a source rate limitation. Secondly, the effect of our proposed solution on the flow stability is highlighted on Figure 7(b), where the end-to-end throughput of the TCP flow is considerably more stable than for the standard 802.11 case.

\section{RELATED WORK}

Multihop wireless networks impose an interesting set of challenges in general [11] and in particular in experimental indoor and outdoor settings [16].

Our work focuses on mesh nodes with a single WiFi card because most mesh networks today are built with 
single cards. Our work therefore contrasts solutions for multi-channel or multi-antenna systems [18], [20], [17].

Our work aims at understanding and addressing challenges for multihop networks at the MAC layer. Our approach therefore differs from related work aimed at MAC layers for single-hop scenario, e.g. [9] and [19].

In [14], the authors also focus on MAC layer performance for multihop mesh networks. However, their approach is based on buffer queue management, while our solution targets MAC layer parameter.

Recent work [12], [15] also discusses the hidden node situation. In [15], the authors focus on the routing instability problem and propose source rate limiting as a solution. Complementary solutions to solve the interflow unfairness are analyzed in [12] through simulation. Our work differs from both these approaches by focusing on the intra-flow behavior and presenting simulation as well as experimental results to support our analysis of the impact of MAC 802.11 backoff policy.

Finally, the methodology of applying flow models from fluid physics has been successfully used, e.g. for vehicular traffic [13]. We are exploiting and combining models from both areas now to model multihop traffic.

\section{CONCLUSiOns}

This paper presents novel insights into the behavior of MAC layer protocols on the performance of a multihop wireless backhaul networks. The detailed understanding of the flow behavior over multiple hops is crucial for end-to-end flow properties and the use of the network capacity. The understanding that the backoff mechanism leads to turbulent flow behavior and thus the above drawbacks is vital for the design and deployment of wireless mesh networks.

Our results are consistent in model, simulations and the experimental evaluation in our testbed. This conclusion is particularly important because the effect of contention is local, i.e. affecting the communication of neighboring TAPs only. However, we show that this local event affects in fact the resource usage of the entire network as well as the end-to-end performance.

The concept of laminar and turbulent flows is a promising approach towards understanding and modeling MAC layer behavior, but it has the potential to be suited for higher layer behavior, such as routing or end-to-end congestion control. In future work, we will continue our study on flow behavior in general, as well as the impact of interacting flow behavior, such as TCP over multihop mesh networks.

\section{REFERENCES}

[1] The Cloud. available at http://www.thecloud.net/.

[2] EarthLink. available at http://www.earthlink.net/.

[3] Freifunk. available at http://freifunk.net/.

[4] MIT Roofnet. available at http://pdos.csail.mit.edu/roofnet/.

[5] IEEE802.11, Part 11: Wireless LAN Medium Access Control (MAC) and Physical Layer (PHY) Specifications, Aug. 1999.

[6] A. Aziz, T. Huehn, R. Karrer, and P. Thiran. Model validation through experimental testbed: the fluid flow example. In Proc. of TridentCom'08, Mar. 2008.

[7] J. Bicket, D. Aguayo, S. Biswas, and R. Morris. Architecture and evaluation of the mit roofnet mesh network. In Proceedings of ACM Mobicom'05, Cologne, Germany, Aug. 2005.

[8] J. Camp, J. Robinson, C. Steger, and E. Knightly. Measurement driven deployment of a two-tier urban mesh access network. In Proc. of ACM MobiSys'06, Uppsala, Sweden, June 2006.

[9] M. Durvy and P. Thiran. A packing approach to compare slotted and non-slotted medium access control. In Proc. of INFOCOM'06, Barcelona, Spain, Apr. 2006.

[10] R. Karrer, I. Matyasovszki, A. Botta, and A. Pescape. Magnets: experiences from deploying a joint research-operational next-generation wireless access network testbed. In Proc. of TridentCom'07, May 2007.

[11] R. Karrer, A. Sabharwal, and E. Knightly. Enabling large-scale wireless broadband: the case for taps. SIGCOMM Comput. Commun. Rev., 34(1):27-32, Jan. 2004.

[12] L. Li and P. A. S. Ward. Structural unfairness in 802.11basedwireless mesh networks. In Proc. of CNSR '07, May 2007.

[13] K. Nagel and M. Schreckenberg. A cellular automaton model for freeway traffic. J. Phys. I France 2, page 2221, Dec. 1992.

[14] N. Nandiraju, D. Nandiraju, D. Cavalcanti, and D. Agrawal. A novel queue management mechanism for improving performance of multihop flows in ieee 802.11s based mesh networks. In Proc. of IPCCC, Apr. 2006.

[15] P. C. $\mathrm{Ng}$ and S. C. Liew. Throughput analysis of IEEE 802.11 multi-hop ad hoc networks. IEEE/ACM Transactions on Networking, 15(2):309-322, Apr. 2007.

[16] K. Papagiannaki, M. Yarvis, and W. S. Conner. Experimental characterization of home wireless networks and design implications. In Proc. of INFOCOM'06, Barcelona, Spain, Apr. 2006.

[17] B. Raman and K. Chebrolu. Design and evaluation of a new MAC protocol for long-distance 802.11 mesh networks. In Proc. of ACM MobiCom'05, Cologne, Germany, Aug. 2005.

[18] S. Roy, A. Das, R. Vijayakumar, H. Alazemi, H. Ma, and E. Alotaibi. Capacity scaling with multiple radios and multiple channels in wireless mesh networks. In Proc. of IEEE WiMesh'05, 2005.

[19] V. A. Siris and G. Stamakis. Optimal cwmin selection for achieving proportional fairness in multi-rate 802.11e wlans: Test-bed implementation and evaluation. In Proc. of IEEE WiNTECH'O6, 2006.

[20] J. Tang, G. Xue, and W. Zhang. Maximum throughput and fair bandwidth allocation in multi-channel wireless mesh networks. In Proc. of INFOCOM'06, Apr. 2006.

[21] A. Tirumala, F. Qin, J. Dugan, J. Ferguson, and K. Gibbs. Iperf: The TCP/UDP bandwidth measurement tool. available at http://dast.nlanr.net/Projects/Iperf/. 